\title{
Gaps in Suicide Assessment and Management Among Accident and Emergency Nurses in Kenyatta National Hospital: a Qualitative Study
}

\author{
Rachel Maina $^{1,2} \cdot$ David Bukusi $^{3} \cdot$ Simon Kahonge Njuguna ${ }^{4} \cdot$ Manasi Kumar $^{5}$ \\ Published online: 5 October 2018 \\ (C) The Author(s) 2018
}

\begin{abstract}
This qualitative study was conducted using key informant interviews with Accident and Emergency and Mental Health staff at Nairobi's Kenyatta National Hospital to examine the current state of suicide assessment and management care at the Department. The interviews probed whether protocols were in place and if the key personnel were aware of these procedures and to what extent evidence-based protocol was used in managing suicidal patients at A\&E. We interviewed eight key informants who rotated in the Accident and Emergency Department and Mental Health Department, and they were interviewed using a semi-structured interview guide. We included clinicians and administrators in addition to the nurses. Using NVivo Pro 11 software, themes and subthemes were derived with the final code system having patient assessment and patient management, as well as protocol and care gaps and further recommendations as the key themes. With regard to patient assessment, all the interviews identified stressful life situations, substance use, and violence as being the dominant presenting complaints in patients with suicidal tendencies who were seen at A\&E. Five out of eight interviews with our key respondents mentioned depressive symptoms as a clinical presentation for suicidal patients. Six out of eight respondents also shared that there were no protocols for assessing and managing suicidal patients with some reporting that they used what they learnt in Nursing and Medical School to offer management in that point of time. Most of our respondents mentioned that clinical management of suicidal patients was mostly done with the use of force to deal with violent and resistant patients. Psychological management was essentially an ex post facto issue left to the very few counselors within the Department. Most of our respondents did not have information on their patients after discharge raising questions about patient safety and well-being. Our analyses of these interviews led us to the conclusion that there were significant gaps in assessment as patients were not routinely screened for suicidal ideation despite suicidal intent and psychological problems being undercurrent issues. Psychological management was sparse with most of it being left to very few staff. Training in suicide assessment for all patients and management we felt was critical in increasing the self-efficacy of nurses in Accident and Emergency Department in handling medical emergencies that are prompted due to mental health issues and suicidal and self-harming behaviors.
\end{abstract}

Keywords Brief suicide assessment · Brief suicide interventions · Self-efficacy · Accident and emergency health care worker · Suicide assessment gaps $\cdot$ Suicide management gaps $\cdot$ Suicide protocols

Electronic supplementary material The online version of this article (https://doi.org/10.1007/s40609-018-0127-7) contains supplementary material, which is available to authorized users.

Rachel Maina

maina.rachel@gmail.com

1 Clinical Psychology, BA Psychology \& Sociology (Nrb), Clinical Psychologist University of Nairobi, Nairobi, Kenya

2 Tilburg University, Tilburg, Netherlands

3 Youth Center KNH, Nairobi, Kenya

4 Mental Health, Ministry of Health, Nairobi, Kenya

5 Department of Psychiatric, University of Nairobi, Nairobi, Kenya

\section{Introduction}

Suicide is a global epidemic which is preventable if people under distress are assessed, diagnosed, and managed in good time. Global estimates of complete suicide stand at almost one million with suicide being the third leading cause of death among 15-44-year-olds and the second among 1519-year-olds (World Health Organization 2012). These estimates are disheartening considering suicide is preventable. Indeed, there are several evidence-based strategies, such as limiting access to lethal weapons, that can curtail suicide (Zalsman et al. 2016). In addition, World Health Organization denotes that suicides are preventable (World 
Health Organization 2014). This cautionary statement becomes more stark as a majority of people who eventually complete suicide (up to one in every five and in other studies up to $83 \%$ ) have been in contact with a health care professional prior to this occurrence (Ahmedani et al. 2014; Luoma et al. 2002). This highlights the importance placed by WHO in primary health workers being regularly educated on assessment, management, and referral as well as support for suicidal individuals who will mostly present with physical symptoms (World Health Organization 2012). The nurse at a hospital's Accident and Emergency Department is the most likely health care professional to be initially in contact with patient presenting with an adverse condition secondary to a suicide attempt. This same nurse is also in a unique position for assessing and preventing suicide attempts. This is because studies have found that some medical conditions are associated with elevated suicide risk, and they include stroke, coronary heart disease, and chronic obstructive pulmonary disease (Webb et al. 2012). Despite this unique positioning of nurses in the Accident and Emergency Department, studies have shown that these nurses are not well equipped to handle cases associated with suicidal tendencies. A study conducted across USA in seven states by Betz et al. (2013) showed that $64-70 \%$ of the nurses lacked confidence in risk assessment skills while 46-56\% lacked counseling skills which are important in treating patients with suicidal tendencies as biomedical model is not enough in management. In a study done by Rutto et al. (2012) in the Kenyatta National Hospital's Accident and Emergency Department, a third of the nurses felt uncomfortable and nervous when attending to patients who had attempted suicide and more than half of them expressed frustration when treating them. Nurses, $38.3 \%$ of them, expressed that a strict treatment method should be used in an attempt to prevent future attempts. Such gaps have informed WHO recommendations on suicide prevention among them training of primary health care workers on screening for suicidal thoughts and plans as well as past deliberate self-harms (World Health Organization 2012). In organizing such kind of training, it is important to elucidate the exact gaps in nurses' knowledge and practice as well as system gaps and barriers. A qualitative study would best suit this form of inquiry as it would explore experiences of working with suicidal patients and attributed meanings. This would eventually lead to easy identification of diverse modifiable outcomes that could be addressed during training as well as systems in place that could be used to support any changes required for implementation of effective suicide prevention protocols. This preliminary report taps into this goal by elucidating suicide assessment and management gaps among Kenyatta National Hospital's A\&E nurses towards improving skills and confidence when assessing patients with suicide risk.

\section{Methods}

\section{Participants and Facility Information}

This qualitative study was conducted among eight key informants who had critical information on how Kenyatta National Hospital A\&E Department runs. These included the Head of the Department in the Mental Health Unit, the Head of the Department in the Accident and Emergency Department, and the nurse who is in charge in the Accident and Emergency Department among other clinicians and nurses identified through snow balling. The Mental Health Department is among the 22 outpatient clinics housed in the hospital, and it usually has different cadres of patients coming in at different days of the week. The child clinic runs on Mondays with an average of 10 first-time patients being booked every Monday and around 20 patients being followed up patients. The adolescent and adult clinics are held every other day with around the same number of patients as the child clinic being seeing everyday though the adolescent clinic tends to encounter more patients. The Accident and Emergency Department is a 24-h service area with patient population varying drastically depending on whether there is a crisis or not and number of referrals seen daily. It is the point of referral for also walk in patients who are assessed and referred to any other 22 outpatient clinics.

\section{Ethical Approval}

The study was reviewed by the Kenyatta National Hospital and University of Nairobi Ethical Review Committee (approval no. P697/11/2015), and the respondents gave written informed consent. The approved protocol included a larger study aimed at improving the self-efficacy of nurses in assessment and management of suicidal patients. The larger study will integrate the training of the A\&E nurses in suicide assessment and management as well as support through a referral system within the clinic.

\section{Measures}

A semi-structured interview guide was used with questions touching on availability of a suicide management protocol and current management practices, as well as gaps and barriers to providing suicide prevention. The guide integrated questions on whether there was a suicide management protocol or not and presenting complaints as well as common management practices. All the participants responded to the same questions. Some of the key domains explored included risk factors, signs and symptoms, forms of intervention, preferred management and accompanying guideline, and management outcomes. 


\section{Procedure}

The key informants were contacted through a phone call with brief explanation of the study being given and a date being set up for the interview. On the interview day, the interviewees gave an informed written consent which also recognized sort permission to record the interviews through an audio recorder. The interviews lasted for 20 to $30 \mathrm{~min}$.

\section{Data Analysis Plan}

The interview data was coded as one single data body and analyzed based on systematic coding using the NVivo Pro 11. We carried out a simple thematic analysis keeping the core ideas around suicidal patients, their care, and the knowledge of nursing staff at A\&E and Mental Health departments. The themes were extracted, and then through a method of triangulation, these ideas were shared with a team of three- $\mathrm{MK}, \mathrm{DB}$, and RM who sifted through the material. RM read all the interviews twice while MK reviewed these once and the triangulation process had all three. One is a clinical psychologist and specialist in implementation science issues in mental health, and DB is a psychiatrist focusing on mental health service integration at $\mathrm{KNH}$.

\section{Results}

Our interview findings fall under three main categories of responses we found (Table 1). They include:

1. Issues involved in patient assessment

2. Current management practices

3. Gaps in assessment and management, as well as preferred recommendations

Each of these themes included subthemes.

Table 1 Distribution of themes

\begin{tabular}{ll}
\hline Theme & Subthemes \\
\hline Issues involved in patient assessment & "Signs of a suicidal patient" \\
& "Methods of committing suicide" \\
& "Risk factors" \\
& "Populations at risk" \\
& "Protocol awareness" \\
Current management practices & "Management approach" \\
& "Types of intervention" \\
& "Challenges" \\
Gaps in assessment and management & "Suicide prevention" \\
as well as preferred & "Community sensitization" \\
recommendations & "Capacity building" \\
& "Facility-level improvement" \\
\hline
\end{tabular}

Issues Involved in Patient Assessment $(N=8)$

\section{Signs of a Suicidal Patient}

All the eight respondents described suicidal thoughts and behaviors common among suicidal patients or those providing reason to believe that there's cause for concern. These include feelings of hopelessness, guilt from mistakes committed, wanting to harm themselves, wishing to die, giving up their possessions (such as money, clothes), complaints of frustrations by close relations, some suddenly quitting their jobs, indulging in abnormal behaviors such as drinking alcohol and/or substance abuse, some isolating themselves/ withdrawing from friends, and losing interest in the common things that they liked. Some patients were reported to be easily agitated by petty things while "they are very violent such that you have actually to tie them down. Others are remorseful" (nurse counselor in A\&E) especially men. Five out of eight respondents mentioned depressive symptoms when describing the clinical presentation of these patients (see Table 2 for theme stratification).

The respondents reported instances of missing out on suicidal symptoms. For instance, one clinician talked about a case who was previously been managed for depression but ended up committing suicide when actually he seemed okay. The interview also revealed history/previous suicide attempts among these patients.

Our respondents shared that when the patients come to the facility, their clinical presentation varies depending on the method used in attempt to commit suicide, and thus, there is a need to have an awareness of different medical complications. These include stab wounds and profuse bleeding, smell characteristic of poisoning, unconsciousness, and in some cases, the patients may be delirious, agitated, or extremely violent realizing that the attempt wasn't successful.

\section{Methods Used to Attempt Suicide}

Among the methods mentioned by our hospital staff interviewees, the most commonly seen were ingestion of poisonous agents (mostly organophosphates such as herbicides), drug overdose, stabs using sharp objects (pangas otherwise known as machetes, knife, etc.), and hanging attempts with the use of rope or clothes. Organophosphates are organic compounds that contain the chemical phosphate prevalently used in insecticides such as fertilizers.

\section{Gender-Based Preference of Suicidal Methods}

The hospital staff also mentioned that the choice of suicide method varied by gender. Men appear to be more determined when committing suicide compared to women as observed in their choice of method and execution. This assertion was 
Table 2 Theme stratification

\begin{tabular}{|c|c|c|}
\hline Themes & Subthemes & Comments (frequency) \\
\hline \multirow[t]{31}{*}{ Patient assessment } & \multirow[t]{15}{*}{ Signs of a suicidal patient } & Hopelessness/despair $(n=2)$ \\
\hline & & Complaints of frustrations $(n=2)$ \\
\hline & & Agitation $(n=1)$ \\
\hline & & Withdrawing from friends and family $(n=2)$ \\
\hline & & Talking of wishing to die/kill themselves $(n=3)$ \\
\hline & & Feelings to harm themselves $(n=1)$ \\
\hline & & Attempted suicide $(n=2)$ \\
\hline & & Stop going to work $(n=3)$ \\
\hline & & Involve in taking drugs and drinking alcohol $(n=2)$ \\
\hline & & Losing interest in common things they liked $(n=1)$ \\
\hline & & Giving out possessions $(n=2)$ \\
\hline & & Depression symptoms $(n=3)$ \\
\hline & & Violent and/or remorseful patients $(n=2)$ \\
\hline & & Physical harm—stab wounds $(n=2)$ \\
\hline & & "... it is very difficult to know, not unless the relatives come and tell you..." $(n=1)$ \\
\hline & \multirow[t]{4}{*}{ Methods of committing suicide } & Organophosphate poisoning $(n=5)$ \\
\hline & & Drug overdose $(n=1)$ \\
\hline & & Stabbing (using pangas, kitchen knives, or razor blades) $(n=2)$ \\
\hline & & Hang by rope $(n=1)$ \\
\hline & \multirow[t]{8}{*}{ Risk factors for suicide } & Loss of job $(n=1)$ \\
\hline & & Loss of family members/relatives $(n=1)$ \\
\hline & & Family issues $(n=1)$ \\
\hline & & Work-related stress $(n=1)$ \\
\hline & & Rape $(n=2)$ \\
\hline & & Relationship issues (left by a loved one) $(n=1)$ \\
\hline & & Unemployment $(n=1)$ \\
\hline & & $\begin{array}{l}\text { "...functional problem; hopelessness and helplessness..." }(n=1) \\
\text { Suicide attempt history }(n=2)\end{array}$ \\
\hline & \multirow[t]{4}{*}{ Populations at risk } & “...suicidal cases, we usually see from 20 s to 30 s, yeah, 35 there..." $(n=1)$ \\
\hline & & "And majority of them unfortunately are men" $(n=2)$ \\
\hline & & People with severe illness $(n=2)$ \\
\hline & & People who have suffered losses (job, family, etc.) $(n=1)$ \\
\hline \multirow[t]{10}{*}{ Patient management } & \multirow[t]{2}{*}{ Protocol awareness } & “...don't have a protocol” $(n=6)$ \\
\hline & & “...have protocol for managing..." (WHO, SOP) $(n=2)$ \\
\hline & \multirow[t]{3}{*}{ Types of intervention } & Psychological support $(n=6)$ \\
\hline & & Gastric lavage $(n=3)$ \\
\hline & & Antidepressants $(n=1)$ \\
\hline & Management approach & $\begin{array}{l}\text { Restraint }(n=1) \\
\text { Sedation }(n=1)\end{array}$ \\
\hline & \multirow[t]{4}{*}{ Outcomes experienced often } & No follow-up $(n=2)$ \\
\hline & & Follow-up done $(n=3)$ \\
\hline & & $\begin{array}{l}\text { "... a co-morbidity with alcohol, you are likely to get worse outcome..." “...out- } \\
\text { comes are not good because these poisonous substances..." }(n=2)\end{array}$ \\
\hline & & $\begin{array}{l}\text { "... a lot of the patients I'm managing are patients who are now controlled..." “... } \\
\text { most of our patients actually walk out alive..." "... some they wake up ..." } \\
(n=3)\end{array}$ \\
\hline \multirow[t]{2}{*}{ Gaps and recommendations } & Staff empowerment & Training $(n=2)$ \\
\hline & $\begin{array}{l}\text { Community sensitization } \\
\text { Facility-level improvement }\end{array}$ & Training in society and institutions $(n=2)$ \\
\hline
\end{tabular}


made clearer when one of our interviewees said "When men really want to commit suicide, they are determined. So you find that if at all this person has taken some drugs they really take them in real quantities and so they come when they are already down but for the ladies sometimes they just want to attract attention. So the substances that they normally take don't even have much effect and when they come, they are not as badly out of control as the men." For instance, a man would ingest an organophosphate which is very poisonous and has a higher probability to kill within a short time frame instead of taking a drug overdose (also see Table 2).

\section{Situational Risk Factors Triggering Suicide}

The hospital staff mentioned a number of psychosocial factors associated with risk of suicide and suicide attempts. They included "...they lose hope because of jobs, those ones who are already educated; they have finished with their colleges. Others it is about the relationship, somebody has been left by the loved ones, others they want to commit suicide because they have lost part of the bodies, others they have lost their relatives, like a person will lose the mother and that is the only hope they had, or even a father but most of them they will complain of losing the mother then they want to commit suicide because they are hopeless..."; finances; chronic or longterm problems such as long periods of unemployment; family issues; problems at school especially among the teenagers; issues related to work; psychological states of acute distress like despair, guilt, and shame; and terminal illnesses such as cancer and HIV infection.

\section{Populations at Risk of Suicide}

At-risk groups emerging from the interviews were people with chronic/terminal illnesses such as HIV-positive patients, patients with history of rape "...patients who are raped, most of the times, they like to commit suicide..." as well as attempted suicide, people with family history of suicide, people with drinking problems, and teenagers/adolescents. One respondent also mentioned that majority of the suicide cases attended to at $\mathrm{KNH}$ range between the age of 20 and 35 years and that most of them are men.

\section{Aspects of Patient Management}

\section{Protocol Awareness}

Six out of eight hospital staff interviewees mentioned that there are no written protocols or SOPs for assessing and managing suicidal patients. They were also not aware of their existence in other health facilities. However, there were two interviewees who confirmed having protocols for managing these patients with one mentioning the WHO protocol as well as standard operating procedures. Considering the eight respondents work in the same facility, what would be the reason behind the contradicting information or a few people knowing protocols and a few not being aware of these? Despite confirming the existence of the protocol, the interviews were quick to point out the fact that effective implementation of these protocols at $\mathrm{KNH}$ is a challenge due to lack of inpatient wards that are secure for the admission of these patients. The following vignette makes this point clearer.

We can't apply it because we don't have a mental health in-patient unit”, “...look at those big windows and look at the security. A hundred patients per ward, with two nurses who are on duty, and there are no security people. Can you manage a suicidal patient?

Most of the interviewees talked of referring these patients to Mathari Teaching and Referral Hospital or private facilities with safe wards after assessing the risk.

All the interviewees believed it was important to have these protocols in place so as to aid health care providers in identifying patients with suicidal thoughts especially for those with no previous training or experience in management of psychiatric patients. The protocols will also standardize the patient management criteria, thus ensuring uniformity irrespective of whether a doctor, a nurse, a counselor, a psychiatrist, or a psychologist is the first to attend to the patient.

\section{Medical Intervention}

Among the major interventions for suicidal patients mentioned include medical interventions (which entail certain procedures such as gastric lavage, a gastrointestinal decontamination procedure for patients who have ingested poison; electroconvulsive therapy (ECT); surgical interventions in cases of stab wounds and "...manage their condition with medications ...").

\section{Limited Psychological Intervention}

This is a management option where the patients are counseled to help them opting for healthy means of coping with the situation other than opting to commit suicide and help them understand that the problem can be solved. Among the responses was an interview that pointed out that they now have a "...psychologist to do the psychotherapy and then if social worker is needed because of, probably this person needs follow up, then maybe home placement..." Family interventions are integrated where the patient's close relatives are informed about the patient's condition to have them support him/her to cope with the situation in a healthy way. 


\section{Management Cascade}

The management approach depends on several factors which mainly the patient's suicidal risk assessment - the magnitude of the suicidal risk, lethality of the means used, and general state of the patient, "...if the patient is in a poor condition you need to manage the condition" (Fig. 1).

The first step as mentioned by all the interviewees was to identify if the client is a suicidal patient/has suicidal tendencies. The patient's clinical presentation (such as depressive symptoms, presence of stab wounds, unconsciousness, and violent patients), history of patient, suicidal attempt report/claims given by the accompanying relative or police, psychological evaluation, or mental examination can be used at this stage.

The next course of action depended on the final diagnosis and patient presentation; the most frequently mentioned suicidal patient diagnoses were poisoning/overdose, stab wounds/cuts, and patients with suicidal tendencies (violent, with suicidal thoughts and ideations, etc.).

For patients who have ingested poison (organophosphate being the most frequently mentioned), “...if they are still alert, not unconscious...," a gastric lavage procedure is done to clean the stomach, after which IV fluids and antidotes are administered to stabilize them and neutralize the poison. These kinds of patients are managed either in ICU or in the ward depending on the severity, "....some they can take a lot of drugs or overdose so most of those cases we treat them in ICU because sometimes they are even not breathing."

Patients with signs of physical harm such as cuts (using machetes, knives, etc.) receive surgical intervention to repair the damage either in the theater or in the wards (can be admitted in ENT or cardiothoracic units or other units depending on their organ damage).

Patients found to have suicidal thoughts/ideations undergo psychological evaluation and mental status examination after which they are either recommended for counseling if the risk of suicide is mild or moderate. For those deemed to be at high risk, they are admitted for psychotherapy and "...to protect him from himself or herself...." For those who considered "...very, very risky...," clinicians refer them for further management at Mathari Teaching and Referral Hospital because KNH lacks safe mental health wards/units (e.g., those diagnosed with mental disorders, those previously treated at Mathari, etc.)

After the patient has been stabilized and considered to be out of danger, they are taken through a limited psychological intervention which is mainly counseling as mentioned by most of the respondents. The counseling is done by nurses, counselors, and psychologists. For some patients, the psychiatrist ensures that the suicidal patients “... are on antidepressants and they are on supportive psychotherapy..." This can be done while the patient is still in hospital and continuous after discharge unless the patient feels okay. The nurse counselor also helps in management as evidenced in the vignette below.

...With counseling. We continue with counseling not less than six to ten sessions for them to come out of whatever that was making them to need to commit suicide ....

Five out of eight interviewees do not have information on their patients after discharge. Counselors are tasked with this role with the aid of social workers.
Fig. 1 Management cascade

Suicide Assessment

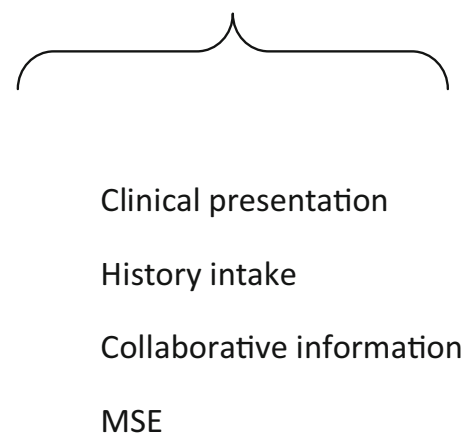

Diagnosis

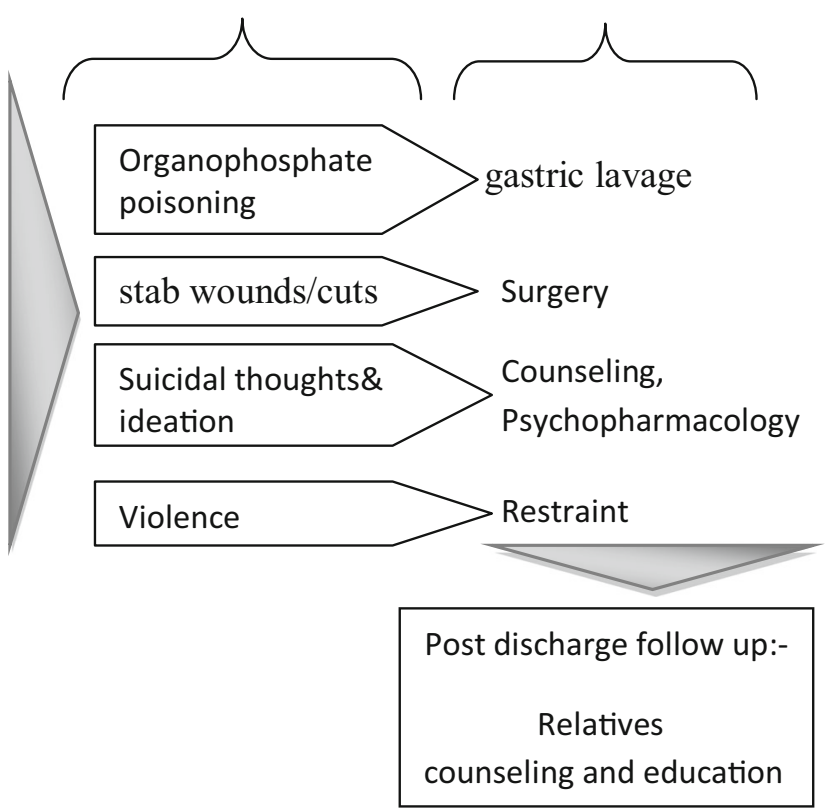


As part of post-discharge management, relatives of certain patients (like rape cases, patients with complaints of family issues, teenagers) are informed and counseled about the patient's condition to assist in supportive psychosocial therapy for better health outcomes.

\section{Outcomes Experienced Often}

Four of the respondents mentioned that most of their patients have good outcomes after care. However, some have poor outcomes like those "...who had other complications, like when they had marital issues and they were drinking." The management outcome also depends on how long the patient takes before coming to the hospital, "...if they take so long, then the outcomes are not good because these poisonous substances would have been absorbed into the system so you find that some of them need to be actually mechanically ventilated $\mathrm{coz}$ they are completely down and normally when it is very, in that comatose, they, most of them don't actually come out of it coz the damage is already done." And availability of psychological support at home after discharge cannot be taken for granted. The following remarks by one of the nurses make this clearer.

...if we let them go out after discharge and there is still not psychological support from home, maybe they now been put as outcasts, there is no body to talk to, the problems are still there with at home the wife or the husband then I don't know how our success is...

\section{Identified System and Facility-Level Gaps}

The lack of safe inpatient wards and dedicated mental health unit was the most frequently mentioned gap. Other identified gaps included lack of suicide assessment and management protocol, lack of training on management of these patients among staff particularly the new health care providers (nurses were mostly mentioned), and low community awareness around suicide. This is better extrapolated in the vignette below.

...not all of us were trained on mental health although most of us, we are counselors. And especially if somebody is new, you have not worked in this department for a long time, you may not identify...

...So our society needs training. So if you start with the nurses then we move outside....

The recommendations given by the interviewees targeted the staff (health care workers), health facilities, and community (see Table 3 for more vignettes along this line). Based on the interviews and feedback from our participants, we felt that for the health worker and health facility staff empowerment through capacity building to equip the health care providers with skills around mental health care and suicide management was important. In this regard, adoption of clear protocols and establishment of a referral system to be used when the health worker alone is unable to manage the patient to guide the next steps were also very important. As pointed out by majority of the interviewees, there needs to be a safe inpatient mental facility/unit for suicidal patients. One interviewee also suggested "...that it should be one of the assessments that should be done to every patient that comes to a hospital. Like the way even we are recommending for driving substance abuse screening, this also should be part of it because when patients present to us, they don't just say, "I want to commit suicide." Until you make that assessment that is when you realize, "Oh this patient has those ideations."”

Community sensitization is important when it comes to suicide cases. As a prevention measure "Educate people on once you have family history of depression, there are certain things you need to look out for and avoid doing coz you are at risk" of suicide and to give psychosocial support ensuring good management outcomes, "the communities accept that we have patients who are like that who can get help in any mental health institution and really just reduce the stigma."

\section{Discussion}

Our study found out that there are gaps in psychosocial management of suicidal patients with most health care providers basing management on clinical presentation without assessing for overt suicidal indicators among patients who do not have suicidal clinical presentations. High index of suspicion for suicidal tendencies is reserved for patients who present with depression and substance use problems as well as those who clearly have had a suicide attempt as shown by overdosing or use of other lethal means for committing suicide. With such a biased assessment, most management involves pharmacological and surgical interventions with psychotherapy and counseling being left in the few hands of counselors integrated within the clinic. There is paucity in training on psychological management even among the counselors within the clinic as they do not have extensive mental health training on suicide management. From our interviewed participants, we understood that patients who do not have overt signs and symptoms for suicide may be bypassed as there are no suicide screening measures put in place. Protocols as well for suicide assessment and management are not integrated; hence, nurses and clinicians tend to rely on what they individually learnt in school which may not be widely shared; hence, management tends to be disintegrated. In addition, as much as the nurses know of risk factors to suicide, this knowledge is not properly integrated when it comes to using the risk factors as decision tools in management. Identifying risk factors could help in deciding whether to admit a patient or offer a brief intervention among 
Table 3 Key informants themes and sample vignettes

Key informants Core themes $\quad$ Vignettes

Mental health specialist (not connected to A\&E)
Patient assessment

Patient management Gaps and recommendations
Vignettes

“...there's co-morbidity with alcohol, you are likely to get worse outcome. Or financial, psychosocial stresses, family stresses that tend to perpetuate the illness then they don't do so well. But if it's just pure depression in a supportive environment with supportive family, they respond better..."

“...the follow up could include now psychotherapy and also include psychiatric review if they are still on medication..."

"...we don't have a mental health in-patient unit..."

"...manage their condition with medications..."

"...We continue with counseling not less than six to ten sessions for them to come out of whatever that was making them to need to commit suicide..."

"...I would recommend is that it should be one of the assessments that should be done to every patient that comes to a hospital. Like the way even we are recommending for driving substance abuse screening, this also should be part of it because when patients present to us, they don't just say, "I want to commit suicide." Until you make that assessment that is when you realize, "Oh this patient has those ideations."..."

“...And they are on antidepressants and they are on supportive psychotherapy. Some are undergoing cognitive behavioral therapy..."

"...If the risk is high, then obviously that's a patient who needs admission and to protect him from himself or herself..."

“...depending on the nature of patients that we receive, some of them in very critical medical conditions. We have cancer cases and many other very, very severe cases. And that moment, some of them or most of them could be having suicidal ideations..."

“...some they can take a lot of drugs or overdose so most of those cases we treat them in ICU because sometimes they are even not breathing..."

"...if the patient is in a poor condition you need to manage the condition first..."

A \& E director

Patient assessment Patient management Gaps and recommendations

"...but the men come in when they are unconscious, majority of them, so you really have to pump out the drug that they took..."

"... Once the medical officer reviews the patient, they may decide to sedate the patient depending on how aggressive the patient is. If the patient is just calm and quiet, they do not give them any medication, they just wait for the counselor to take charge. But for the aggressive patients, we usually sedate them at least to calm them down as they wait for the psychiatrist on call to come..."

“...suicidal cases, we usually see from 20 s to 30 s, yeah, 35 there. And majority of them unfortunately are men..."

"...His family has been going through this with him, but they have never told us...."

"...Maybe we can get those protocols. We need to sit down with a team from department of mental health. Come up with protocols, and be able to put them on our notice boards..."

"...I do not know where we involve police, I do not know coz now like suicide is a police case but there is no formal channel of reporting, at least in A \& E, so I do not know..."

"...I do not know whether we do follow them up to see if they went back and took more medication and died in the process or if they changed as a result of our management here..."

"...But if we let them go out after discharge and there is still not psychological support from home..."

“...psychologist to do the psychotherapy..."

"...raped, most of the times, they like to commit suicide..."

Nurse counselor in A\&E Patient assessment

"Some may come when they are very violent such that you have actually to tie them down. Others Patient management are remorseful..."

Gaps and

recommendations

"...There are some who do well and it depends on how long this person had taken before they come to the hospital if they take so long, then the outcomes are not good because these poisonous substances would have been absorbed into the system so you find that some of them need to be actually mechanically ventilated coz they are completely down and normally when it is very, in that comatose, they, most of them do not actually come out of it coz the damage is already done..."

“...lose hope because of jobs, those ones who are already educated, they have finished with their colleges. Others it is about the relationship, somebody has been left by the loved ones, others they want to commit suicide because they have lost part of the bodies, others they have lost their relatives..."

“...When men really want to commit suicide, they are determined. So you find that if at all this person has taken some drugs they really take them in real quantities and so they come when they are already down but for the ladies sometimes they just want to attract attention. So the substances that they normally take do not even have much effect and when they come, they are not as badly out of control as the men..." 
other significant measures in management of a patient with suicide risk. Lethal means of suicide attempt such as drinking harmful chemicals and the use of sharp objects such as knives were common presenting symptoms. Due to such presenting complaints and injuries, Accident and Emergency Department staff tend to attend to people in complex risk and multiple injury contexts. The task of A\&E Department becomes challenging when they see patients present with other conditions that have underlying suicidal tendencies and ideation, and the risk is that without a rigorous training and protocol set in place, these may not be easily captured. Considering one in five deaths by suicide is likely to have had a history of a visit to an emergency setting, it is important to have a high index of suspicion and routine mental health and suicide screen for everyone who attends an emergency setting and effective interventions integrated into normal medical management.

As Betz et al. (2013) have shown, our study also found that nurses and clinicians primarily used the biomedical model for managing suicidal patients as they lack counseling skills which are important in treating patients with suicidal tendencies. Our study also found that they preferred restrictive and strict treatment methods with patients who were violent and suicidal at the same time. This observation was confirmed by a study carried out by Rutto et al. (2012) who found oppressive and harsh practices used to manage violent and uncooperative patients. However, the reason behind these preferred modes of management has not been investigated. With this study, this outcome has been well defined.

Poor knowledge on suicide assessment and management likely curtails measures of reducing completed suicides. Educating clinicians has been found to be among the most significant determinants in reducing suicide rates (Mann et al. 2005). Indeed, a study by Jenkins et al. (2015) recommended training of frontline health workers in a bid to capture people with suicidal ideations early enough. The nurses at Kenyatta National Hospital's Accident and Emergency center lack enough knowledge and experience in dealing with suicidal patients, hence lack confidence in attending to these patients. With this acknowledgement and our study findings, it is vital to improve knowledge in the assessment and management of suicidal tendencies and more so among staff who are more likely to be frontline health care givers. The ISSAM project will integrate such kind of training where nurses in the A\&E will be taught on how to carry out a brief assessment and use risk factors as decision-making tools as well as integrate a brief and effective psychological intervention.

This study was not without limitations. It took place in a clinical setting and hence results and implications may not be generalized to a community setting. However, the objectives of this study could only be clearly extrapolated using a clinical setting with participants who may display certain bias in the protocol knowledge despite being congruent and well attuned to procedures in the clinical context. Moreover, our study findings were comparable to other studies carried out in a similar setting.

\section{Conclusions}

Suicide assessment and management knowledge including psychosocial management are important in the management of patients in the Accident and Emergency Department. Lack of this knowledge and protocols to guide management impends effective and efficient identification of patients at risk. It is important for nurses, who are the front liners in management at the Accident and Emergency Department to have a high index of suspicion for every patient they attend to as well as be in a position to offer psychological support.

\section{Compliance with Ethical Standards}

Conflict of Interest The authors declare that they have no conflict of interest.

Ethical Approval This study has been approved by the Kenyatta National Hospital/University of Nairobi Institutional Review Board under the project "Improving self-efficacy on suicide assessment and management through training of accident and emergency nurses in Kenyatta National Hospital (The ISSAM Project) (P697/11/2015).”

Open Access This article is distributed under the terms of the Creative Commons Attribution 4.0 International License (http:// creativecommons.org/licenses/by/4.0/), which permits unrestricted use, distribution, and reproduction in any medium, provided you give appropriate credit to the original author(s) and the source, provide a link to the Creative Commons license, and indicate if changes were made.

\section{References}

Ahmedani, B.K., Simon, G.E., Stewart, C., Beck, A., Waitzfelder, B.E., Rossom, R., ... Solberg, L.I. (2014). Health care contacts in the year before suicide death. Journal of General Internal Medicine, 29(6), 870-877. https://doi.org/10.1007/s11606-014-2767-3.

Betz, M.E., Sullivan, A.F., Manton, A.P., Espinola, J.A., Miller, I., Camargo, C.A., \& Boudreaux, E.D. (2013). Knowledge, attitudes, and practices of emergency department providers in the care of suicidal patients. Depression and Anxiety, 1012(October 2012), n/a-n/a. https://doi.org/10.1002/da.22071.

Jenkins, R., Othieno, C., Omollo, R., Ongeri, L., Sifuna, L., Ongecha, M., ... Ogutu B. (2015). Tedium vitae, death wishes, suicidal ideation and attempts in Kenya-prevalence and risk factors. Biomed Central Public Health, 15(1)759. https://doi.org/10.1186/s12889-015-2089-3.

Luoma, J. B., Martin, C. E., \& Pearson, J. L. (2002). Contact with mental health and primary care providers before suicide: a review of the evidence. American Journal of Psychiatry, 159(6), 909-916. https:// doi.org/10.1176/appi.ajp.159.6.909.

Mann, J. J., Apter, A., Bertolote, J., Beautrais, A., Currier, D., Haas, A., et al. (2005). Suicide prevention strategies: a systematic review. JAMA : The Journal of the American Medical Association, 294(16), 2064-2074. https://doi.org/10.1001/jama.294.16.2064.

Rutto, J., Mwaura, J., Chepchirchir, A., \& Odero, T. (2012). Nurse's knowledge, attitude and practice on the initial management of acute 
poisoning among adult casualties: study at Kenyatta National Hospital, Kenya. Open Journal of Nursing, 2(03), 149-156. https://doi.org/10.4236/ojn.2012.23023.

Webb, R. T., Kontopantelis, E., Doran, T., Qin, P., Creed, F., \& Kapur, N. (2012). Suicide risk in primary care patients with major physical diseases. Archives of General Psychiatry, 69(3), 256-264. https:// doi.org/10.1001/jamapsychiatry.2014.3039.

World Health Organization. (2012). Public health action for the prevention of suicide: a framework. Geneva: World Health Organization ISBN 9789241503570.
World Health Organization. (2014). Preventing suicide: a global imperative. Geneva: World Health Organization ISBN 9789241564779.

Zalsman, G., Hawton, K., Wasserman, D., Heeringen, K.V., Arensman, E., Sarchiapone, M., ... Zohar, J. (2016). Suicide prevention strategies revisited: 10-year systematic review. The Lancet Psychiatry, 3(7), 646-659. https://doi.org/10.1016/s2215-0366(16)30030-x. 